\title{
Boson fusion and Higgs production at the CERN LHC in six fermion final states with one charged lepton pair
}

\author{
Elena Accomando, ${ }^{*}$ Alessandro Ballestrero, ${ }^{\dagger}$ Aissa Belhouari, ${ }^{\ddagger}$ and Ezio Maina ${ }^{\S}$ \\ INFN, Sezione di Torino and Dipartimento di Fisica Teorica, Università di Torino, Via Giuria 1, 10125 Torino, Italy
}

(Received 28 September 2006; published 20 June 2007)

\begin{abstract}
Boson boson scattering and Higgs production in boson boson fusion will be actively investigated at the CERN LHC. We have performed a parton level study of all processes of the type $q_{1} q_{2} \rightarrow q_{3} q_{4} q_{5} q_{6} l^{+} l^{-}$ using for the first time a full fledged six fermion Monte Carlo event generator which employs exact matrix elements at $\mathcal{O}\left(\alpha_{e m}^{6}\right)$. We have examined Higgs production in vector boson fusion followed by the decay chain $H \rightarrow Z Z \rightarrow l^{+} l^{-} j j$, including exactly all electroweak irreducible backgrounds. In the high mass region we have compared the case of a relatively light Higgs with the no-Higgs results. The integrated cross section for the latter case is more than twice that in the former for a minimum invariant mass of the $\mathrm{ZV}$ pair of about $800 \mathrm{GeV}$. We find, in a preliminary analysis at parton level, that, summing up the muon and the electron channels, about 25 events are expected in the light Higgs case for $L=100 \mathrm{fb}^{-1}$.
\end{abstract}

DOI: 10.1103/PhysRevD.75.113006

PACS numbers: $12.15 .-\mathrm{y}$

\section{INTRODUCTION}

The standard model (SM) provides the simplest and most economical explanation of electroweak symmetry breaking (EWSB). Detailed reviews and extensive bibliographies can be found in Refs. [1-4]. The only missing ingredient is the Higgs boson. The fit of the SM to precision EW data currently gives an upper limit on the Higgs mass of about $200 \mathrm{GeV}$ [5] while direct searches have established a 95\% C.L. lower bound, $M(H)>$ 114.4 GeV [6].

In the SM the Higgs is essential to the renormalizability of the theory and is also crucial to ensure that perturbative unitarity bounds are not violated in high energy reactions. Scattering processes between longitudinally polarized vector bosons $\left(\mathrm{V}_{L}\right)$ are particularly sensitive in this regard. Without a Higgs, the $\mathrm{V}_{L}$ 's interact strongly at high energy, violating perturbative unitarity at about $1 \mathrm{TeV}$ [7]. If, on the contrary, a relatively light Higgs exists, then they are weakly coupled at all energies. In the strong scattering case, one is led to expect the presence of resonances in $\mathrm{V}_{L} \mathrm{~V}_{L}$ interactions. Unfortunately, the mass, spin, and even number of these resonances are not uniquely determined [8,9]. If a Higgs particle is discovered, it will nonetheless be necessary to verify that indeed longitudinally polarized vector bosons are weakly coupled at high energy by studying boson boson scattering in full detail.

At the CERN LHC no beam of on shell EW bosons will be available. Incoming quarks will emit spacelike virtual bosons which will then scatter among themselves and finally decay. These processes have been scrutinized for a long time, from the pioneering works in [10,11], which address boson boson scattering on a general ground, to the

\footnotetext{
*accomand@to.infn.it

†ballestr@to.infn.it

belhouar@to.infn.it

§maina@to.infn.it
}

more recent papers in $[12,13]$, which focus on the extraction of signals of vector boson scattering at the LHC. All previous studies of boson boson scattering at high energy hadron colliders, with the exception, to our knowledge, of $[14,15]$, have resorted to some approximation, either the equivalent vector boson approximation (EVBA) [16], or a production times decay approach, supplementing a calculation of

$$
q_{1} q_{2} \rightarrow q_{3} q_{4} V_{1} V_{2}
$$

processes with the, typically on shell, decay of the two vector bosons. There are, however, issues that cannot be tackled without a full six fermion calculation like exact spin correlations between the decays of different heavy particles, the effect of the nonresonant background, the relevance of the offshellness of boson decays, and the question of interferences between different subamplitudes. Without a complete calculation it will be impossible to determine the accuracy of approximate results. In Ref. [14] this issue was discussed at length, showing differences of the order of $10 \%-20 \%$ in some important regions of phase space. The reliability of the EVBA in the context of vector boson scattering has been critically examined in [17].

Recently PHASE, a full fledged six fermion Monte Carlo, has become available [18]. It describes at $\mathcal{O}\left(\alpha_{e m}^{6}\right)$, using exact tree-level matrix elements, all processes of the form $P P \rightarrow q_{1} q_{2} \rightarrow q_{3} q_{4} q_{5} q_{6} l \nu$ [where $q_{i}$ stands for a generic (anti)quark] which can take place at the LHC $[14,19]$. The range of interesting reactions is however much wider. Processes in which both vector bosons decay leptonically have been extensively studied both for Higgs detection and for boson boson scattering and top physics. Besides, in order to obtain a full coverage of all semileptonic processes, it is necessary to include all reactions with a charged lepton pair in the final state. This has required the calculation of additional amplitudes and an extensive improvement of the routines which pilot the integration 
and the generation of unweighted events. The result is a new code called PHANTOM [20] which, at present, includes all processes with six fermions in the final state at $\mathcal{O}\left(\alpha_{e m}^{6}\right)$,

$$
P P \rightarrow q_{1} q_{2} \rightarrow f_{1} f_{2} f_{3} f_{4} f_{5} f_{6} .
$$

The accuracy of tree-level calculations can be sensibly improved. PHASE is being continued also in this direction. In particular, electroweak corrections have proved to be sizable especially for processes involving the Higgs boson; see for instance Ref. [21]. A new code PHAST_NLO [22] will address $O\left(\alpha_{e m}\right)$ electroweak radiative effects in six fermion physics. Both PHANTOM and PHAST_NLO are based on the methods of Refs. [23,24] and adopt the iterativeadaptive multichannel strategy developed in [18]. In the following we present results obtained with PHANTOM.

PHANTOM is an example of a dedicated event generator which describes a predefined set of reactions striving for maximum speed and efficiency. Other recent examples of dedicated programs for LHC physics are ALPGEN [25] and TOPREX [26]. The complementary approach is given by multipurpose programs for the automatic generation of any user-specified parton level process. The following codes for multiparton production are available: AMEGIC-SHERPA [27], COMPHEP [28], GRACE-GR@PPA [29], MADEVENT [30], PHEGAS\&HELAC [31], O'MEGA\&WHIZARD [32].

Recently the $\mathcal{O}\left(\alpha_{e m}^{6} \alpha_{s}\right)$ QCD corrections to $q_{1} q_{2} \rightarrow$ $q_{3} q_{4} e^{+} \nu_{e} \mu^{-} \bar{\nu}_{\mu}$ have been computed in [33] and those to $q_{1} q_{2} \rightarrow q_{3} q_{4} e^{+} e^{-} \mu^{+} \mu^{-}$and $q_{1} q_{2} \rightarrow q_{3} q_{4} e^{+} e^{-} \nu_{\mu} \bar{\nu}_{\mu}$ in [34]. They turn out to be modest, changing the total cross sections by less than $10 \%$. The smallness of QCD corrections is related to color conservation which forbids gluon exchange between quark lines, up to highly suppressed contributions generated by the exchange of identical quarks. The same applies to QCD corrections to $q_{1} q_{2} \rightarrow$ $q_{3} q_{4} q_{5} q_{6} l^{+} l^{-}$which however do include a larger number of terms which could result in larger corrections. It should be pointed out that QCD effects are not limited to NLO corrections. In the context of vector boson pair production $P P \rightarrow W W$, gluon initiated $\mathcal{O}\left(\alpha_{s}^{2}\right)$ processes $g g \rightarrow W W$, proceeding through a quark loop, have been shown to be a sizable correction to the tree-level reactions $q \bar{q} \rightarrow W W$ when realistic Higgs search cuts are imposed [35]. On the other hand, QCD $W W j j$ production represents only a small background to Higgs searches in vector boson fusion [36].

Since in addition to VV scattering many other subprocesses are, in general, present in the full set of diagrams, as partially shown in Figs. 1-3, it is not a trivial task to

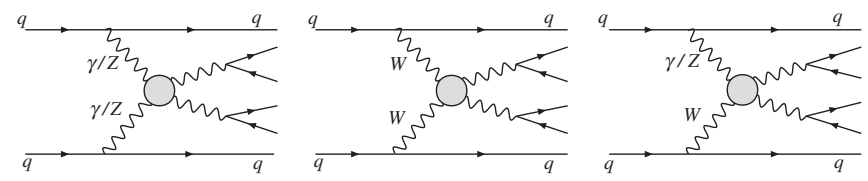

FIG. 1. Vector boson fusion processes.

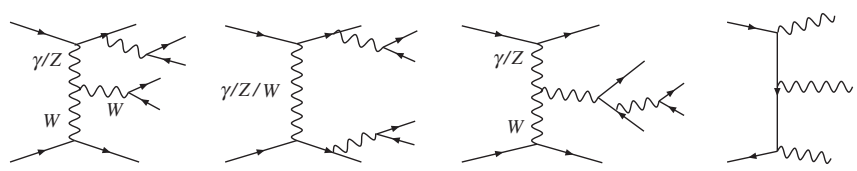

FIG. 2. Examples of EW irreducible background to vector boson scattering processes.
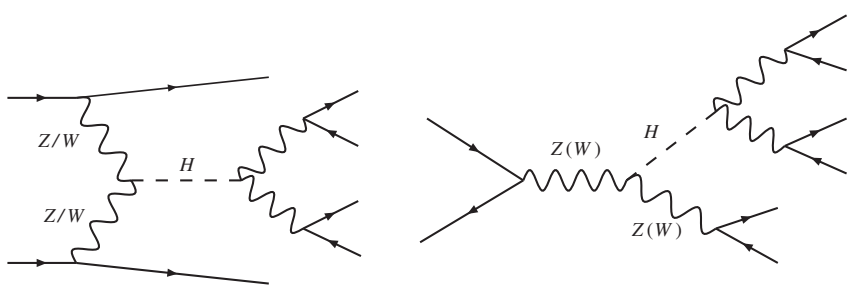

FIG. 3. Higgs boson production via vector boson fusion and Higgsstrahlung.

separate boson boson scattering from the EW irreducible background. In practice, one has to deal also with other types of background to which QCD interactions contribute, but which however do not include any boson boson scattering term. We will refer to these processes as QCD background even though, in general, they will be a mixture of QCD and EW interactions. In this paper we are neglecting QCD backgrounds. It is clear that obtaining a good signal over EW irreducible background ratio is a prerequisite to any attempt at dealing with the QCD one.

We are aware that much is still needed to obtain a thoroughly realistic assessment of the observability of these processes. Only a complete study including $4 j l^{+} l^{-}$ at $\mathcal{O}\left(\alpha_{e m}^{4} \alpha_{s}^{2}\right)$ and $\mathcal{O}\left(\alpha_{e m}^{2} \alpha_{s}^{4}\right)$ together with full detector simulation will be able to say the final word. In the meantime it is important that the tools available for simulation are sharpened as much as possible and that the viability of such analyses is demonstrated at $\mathcal{O}\left(\alpha_{e m}^{6}\right)$ which includes all signal contributions. In our opinion, the results presented in the following and the event generator used to produce them represent a step forward in this direction.

\section{CLASSIFICATION AND CALCULATION}

For a complete analysis one needs to include all processes which contribute to final states with one charged lepton pair. Taking into account one lepton type, charge conjugation, and the symmetry between the first and second quark families, the number of reactions can be reduced to 135. A given reaction, its charge conjugate, the ones related by family exchange and those obtained with the product of the two transformations can be indeed described by the same matrix element; they differ by the convolution with parton distribution functions (PDF). All processes which share the same total particle content, with all eight partons taken to be outgoing, can be described by a single master amplitude. As a consequence, all reactions can be 
TABLE I. Classification of $p p \rightarrow q q^{\prime} \rightarrow 4 q+l^{+} l^{-}$processes. The first column shows the group list, the second the process type as discussed in the text, and the third the corresponding number of diagrams.

\begin{tabular}{|c|c|c|c|c|c|}
\hline Group & Type & Diagrams & Group & Type & Diagrams \\
\hline$u \bar{u} c \bar{c} b \bar{b} l^{-} l^{+}$ & $4 Z$ & 615 & $d \bar{d} s \bar{s} b \bar{b} l^{-} l^{+}$ & $4 Z$ & 615 \\
\hline$u \bar{u} u \bar{u} b \bar{b} l^{-} l^{+}$ & $4 Z$ & 1230 & $u \bar{u} u \bar{u} u \bar{u} l^{-} l^{+}$ & $4 Z$ & 3474 \\
\hline$u \bar{u} u \bar{u} s \bar{s} l^{-} l^{+}$ & $4 Z$ & 1158 & $u \bar{u} u \bar{u} c \bar{c} l^{-} l^{+}$ & $4 Z$ & 1158 \\
\hline$u \bar{u} b \bar{b} b \bar{b} l^{-} l^{+}$ & $4 Z$ & 1606 & $u \bar{u} c \bar{c} d \bar{d} l^{-} l^{+}$ & $4 Z+2 W 2 Z$ & 821 \\
\hline$u \bar{u} s \bar{s} s \bar{s} l_{-}^{-} l^{+}$ & $4 Z$ & 1158 & $u \bar{u} u \bar{u} d \bar{d} l_{-}^{-} l^{+}$ & $4 Z+2 W 2 Z$ & 2126 \\
\hline$u \bar{u} s \bar{s} b \bar{b} l^{-} l^{+}$ & $4 Z$ & 615 & $u \bar{u} d \bar{d} b \bar{b} l^{-} l^{+}$ & $4 Z+2 W 2 Z$ & 880 \\
\hline$d \bar{d} d \bar{d} d \bar{d} l^{-} l^{+}$ & $4 Z$ & 3474 & $u \bar{u} d \bar{d} s \bar{s} l^{-} l^{+}$ & $4 Z+2 W 2 Z$ & 821 \\
\hline$b \bar{b} b \bar{b} b \bar{b} l^{-} l^{+}$ & $4 Z$ & 7506 & $u \bar{u} d \bar{d} d \bar{d} l^{-} l^{+}$ & $4 Z+2 W 2 Z$ & 2126 \\
\hline$d \bar{d} d \bar{d} s \bar{s} l_{-}^{-} l^{+}$ & $4 Z$ & 1158 & $u \bar{d} s \bar{c} u \bar{u} l^{-} l^{+}$ & $2 W 2 Z$ & 484 \\
\hline$d \bar{d} d \bar{d} b \bar{b} l^{-} l^{+}$ & $4 Z$ & 1230 & $u \bar{d} s \bar{c} d \bar{d} l^{-} l^{+}$ & $2 W 2 Z$ & 484 \\
\hline$d \bar{d} b \bar{b} b \bar{b} l^{-} l^{+}$ & $4 Z$ & 1606 & $u \bar{d} s \bar{c} b \bar{b} l^{-} l^{+}$ & $2 W 2 Z$ & 265 \\
\hline
\end{tabular}

classified into 22 groups which are enumerated in Table I. By selecting two initial quarks in each particle group, one obtains all possible processes.

The calculation can be further simplified examining more closely the full set of Feynman diagrams. In some processes, fermions can be paired only into neutral currents (4Z), while in other cases they can form two charged and two neutral currents $(2 Z 2 W)$. Mixed processes are described by a combination of the two sets $(2 Z 2 W+4 Z)$.

The $4 Z$ amplitude was not previously available. The three basic topologies in which the Feynman diagrams appearing in the $4 Z$ amplitude can be classified are shown in Fig. 4. The numbers $N 4 / N 3 / N 2 / N 1 / N 0$ under each topology indicate the number of Feynman diagrams described by this topology if $4 / 3 / 2 / 1 / 0$ fermion pairs are taken to be massive, namely, to have nonzero interaction with the Higgs boson. The flavor of all four fermion pairs are assumed to be different. The complete set of diagrams is eventually generated by exchange of identical particles.

Each rectangle on both sides of the central boson topology is the sum of several subdiagrams representing all

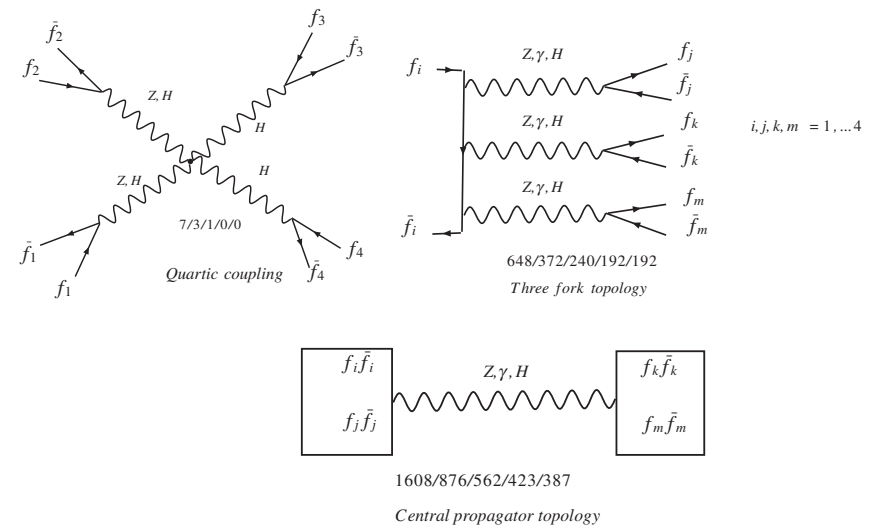

FIG. 4. Fundamental topologies associated with $4 Z$ processes. Total number of diagrams 2263/1251/803/615/579.

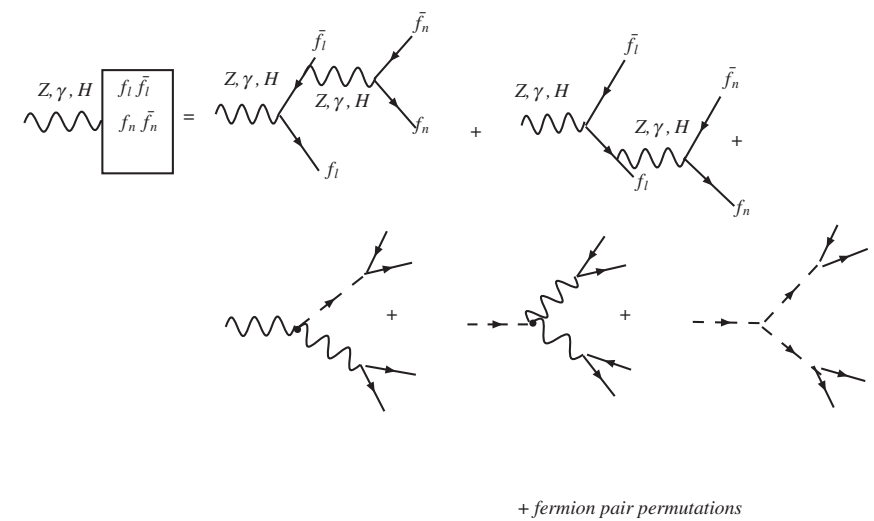

FIG. 5. Decays of the off shell neutral bosons $Z, \gamma, H$.

possible decays of an off shell $Z, \gamma$, or Higgs boson to four outgoing particles as shown in Fig. 5. These sets of subdiagrams are evaluated only once, with a substantial efficiency gain, and then combined together in the end.

\section{PHYSICAL PROCESSES}

Boson boson scattering and Higgs production in boson boson fusion produce intermediate states with two bosons and two quarks as shown in Fig. 1. In this study we have only considered final states in which one $Z$ boson decays leptonically to $\mu^{+} \mu^{-}$and the other (either $Z$ or $W$ ) hadronically. If both bosons decay hadronically the signal cannot be distinguished from the QCD background whose cross section is much larger. Final states where both vectors decay leptonically have a smaller rate and have been left for future studies.

A number of event samples representative of all possible processes of the form $P P \rightarrow q_{1} q_{2} \rightarrow q_{3} q_{4} q_{5} q_{6} l^{+} l^{-}$have been produced with PHANTOM. In order to comply with typical acceptance and trigger requirements, the cuts in Table II have been applied. The acceptance cuts are standard. The wide separation in $\eta$ between the two tag quarks is a well-established technique for separating the scattering signal from the background [11-13]. We have imposed a minimum invariant mass cut of $60 \mathrm{GeV}$ on jet pairs rather

TABLE II. Standard acceptance cuts applied in all results. Any pair of colored fermions must have mass larger than $60 \mathrm{GeV}$. $\Delta \eta$ (tag quarks) is the absolute value of the difference in pseudorapidity between the two tag quarks.

\begin{tabular}{c}
\hline \hline $\mathrm{p}_{T}$ (lepton) $>20 \mathrm{GeV}$ \\
\hline $\mid \eta$ (lepton) $\mid<3$ \\
$\mathrm{E}($ quark $)>30 \mathrm{GeV}$ \\
$\mathrm{p}_{T}($ quark $)>20 \mathrm{GeV}$ \\
$\mid \eta($ quark $) \mid<5$ \\
$\mathrm{M}\left(l^{+} l^{-}\right)>20 \mathrm{GeV}$ \\
$\mathrm{M}($ qq $)>60 \mathrm{GeV}$ \\
$\Delta \eta($ tag quarks $)>3.8$ \\
\hline
\end{tabular}


than requiring a minimum $\Delta R$ separation. It is well known that at large $p_{T}$ the two jets from the hadronic decay of a $W$ or $Z$ boson tend to coalesce. This issue has been discussed, for instance, at length in the ATLAS TDR [3] in connection with the observability of a heavy Higgs decaying to a $W W$ pair, where a number of jet finding schemes have been studied. We defer to Sec. III B a discussion of the effects of a separation in $\Delta R$ for the class of processes under consideration. It should be kept in mind that selection procedures can be optimized in different ways for different analyses and that such optimization has to be performed at hadron level rather than at the more primitive parton stage we are discussing here.

In most of this paper, since we are mainly concerned with extracting signals of boson boson scattering from the irreducible background, we select events using flavor information, which will be unavailable in actual analyses, for the identification of vector bosons and top quarks. In Sec. III B we show that our results are not substantially modified if one adopts a more realistic procedure entirely based on invariant mass cuts.

We have used the CTEQ5L [37] PDF set with scale

$$
Q^{2}=M_{W}^{2}+\frac{1}{6} \sum_{i=1}^{6} p_{T i}^{2}
$$

where $p_{T i}$ denotes the transverse momentum of the $i$ th final state particle.

Many subprocesses (i.e. $Z W \rightarrow Z W, W W \rightarrow Z Z, Z Z \rightarrow$ $Z Z, q b \rightarrow q t V$ ) will, in general, contribute to a specific six fermion reaction. $t \bar{t}$ processes will not contribute to the $4 q l^{+} l^{-}$channels but single top production with an additional neutral boson emission will be present.

It is impossible to separate and compute individually the cross section due to a single subprocess, since there are large interference effects between the different contributions. We can however select all complete $2 \rightarrow 6$ processes which include a specific set of subdiagrams. For instance, $Z W \rightarrow Z W$ with on shell bosons is described by four Feynman diagrams. These same diagrams, with the two incoming external vector bosons connected to the initial fermion lines and the two final ones connected to their decay products, constitute the $Z W \rightarrow Z W$ set of $2 \rightarrow 6$ diagrams. Several sets can contribute to a single process and therefore the same process can appear in different groups; for example, $u d \rightarrow u d \mu^{+} \mu^{-} b \bar{b}$ will be included in both the $W W \rightarrow Z Z$ and $Z Z \rightarrow Z Z$ groups. As a consequence of this multiple counting, the total cross section is smaller than the sum of the cross sections for the various groups. The upper part of Fig. 6 shows the invariant mass distribution of the two most central quarks (when ordered in pseudorapidity $\eta$ ) and of the two leptons for all reactions which contain the different subprocesses as well as the distribution for the complete set of processes. We assumed $M(H)=150 \mathrm{GeV}$. Notice that the Higgs peak is present in the $Z W \rightarrow Z W$ curve. This is due to processes, like, for
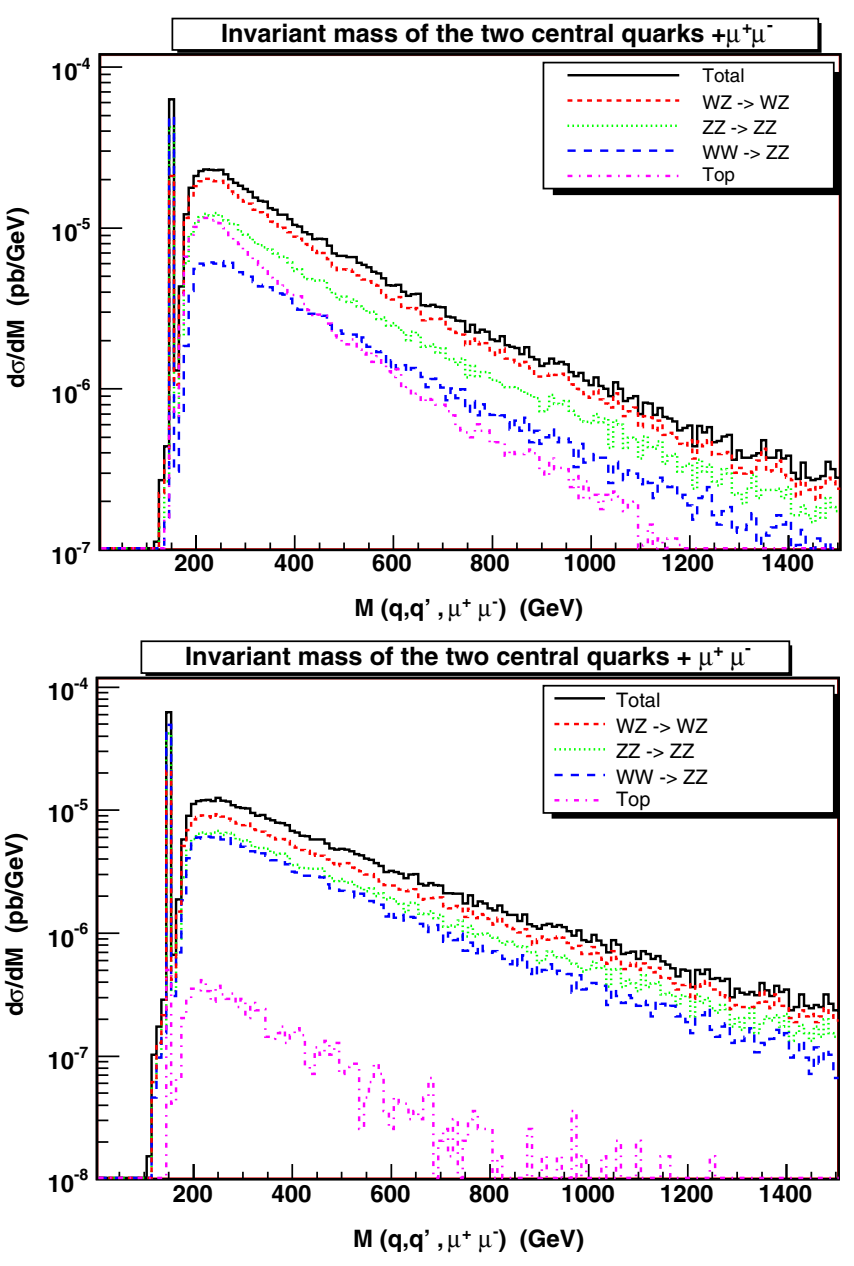

FIG. 6 (color online). Invariant mass distribution of the two charged leptons and the two most central quarks, for different sets of processes. The upper plot includes the set of cuts described in the text. In the lower plot a further cut for vetoing top production is applied.

instance, $u \bar{d} \rightarrow u \bar{d} d \bar{d} \mu^{+} \mu^{-}$, which in addition to the $Z W \rightarrow Z W$ set of diagrams (e.g. $u \bar{d} \rightarrow d \bar{d} W Z$ ) include also diagrams describing Higgs production in the $s$ channel (e.g. $u \bar{d} \rightarrow u \bar{d} Z Z$ ).

The group comprising top diagrams has a large cross section. The lower part of Fig. 6 shows the same distributions after top subtraction. Top candidates are identified requiring a $b$ quark and two other quarks in the final state of the right flavor combination to be produced in a $W$ decay, with a total invariant mass between 160 and $190 \mathrm{GeV}$.

If no Higgs is present, all SM scattering processes between on shell weak vector bosons grow linearly with the center of mass energy squared, with the exception of $Z Z \rightarrow Z Z$ which in this case is zero. This behavior is in agreement with the low energy theorem (LET) [38]. The lower part of Fig. 6 shows that the $Z Z \rightarrow Z Z$ component is relatively small compared with the total distribution and as a consequence does not represent too serious a background to searches for new physics signals. The group including 
TABLE III. Total cross section for $p p \rightarrow q q^{\prime} \rightarrow 4 q+$ $\mu^{+} m u^{-}$with the standard acceptance cuts in Table II for different Higgs masses.

\begin{tabular}{cc}
\hline \hline$M_{H}$ & Cross section $(\mathrm{fb})$ \\
\hline $150 \mathrm{GeV}$ & $7.33 \pm 0.02$ \\
$200 \mathrm{GeV}$ & $13.67 \pm 0.03$ \\
$500 \mathrm{GeV}$ & $9.89 \pm 0.02$ \\
No Higgs & $7.34 \pm 0.02$ \\
\hline \hline
\end{tabular}

TABLE IV. Scale dependence of the total cross section for $p p \rightarrow q q^{\prime} \rightarrow 4 q+\mu^{+} m u^{-}$with the standard acceptance cuts in Table II for two Higgs scenarios. $Q$ is defined in Eq. (3).

\begin{tabular}{crrr}
\hline \hline$M_{H}$ & \multicolumn{1}{c}{$Q / 2$} & \multicolumn{1}{c}{$Q$} & \multicolumn{1}{c}{$2 Q$} \\
\hline $200 \mathrm{GeV}$ & $14.21 \pm 0.02$ & $13.67 \pm 0.03$ & $13.11 \pm 0.02$ \\
No Higgs & $7.57 \pm 0.02$ & $7.34 \pm 0.02$ & $7.01 \pm 0.02$ \\
\hline \hline
\end{tabular}

the $Z W \rightarrow Z W$ set of diagrams gives the largest contribution.

In Table III we present the total cross section for $p p \rightarrow$ $q q^{\prime} \rightarrow 4 q+\mu^{+} m u^{-}$with the standard acceptance cuts in Table II for different Higgs masses. In Table IV we show the scale dependence of the total cross section for two Higgs scenarios, adopting the usual recipe of varying the scale by a factor of 2 in either direction. This leads to a $4 \%$ difference with respect to the central value.

\section{A. Higgs production and its complete EW background in PHANTOM}

PHANTOM is capable of simulating Higgs production in $V V$ fusion together with all its EW irreducible background for all channels and for any Higgs mass and may be particularly useful in the intermediate mass range, below the $W W$ threshold, where the production times decay approach cannot be used. Its improved treatment of the EW sector needs to be complemented by an accurate description of QCD dominated backgrounds and of the effects of QCD NLO corrections.

Higgs production in $V V$ fusion followed by Higgs decay to $W W$ or $Z Z$ is the second most abundant production channel over almost the full range of Higgs masses which will be explored at the LHC. It is regarded as the channel with the highest statistical significance for an intermediate mass Higgs [36,39]. Previous analyses have focused mainly on the $W W$ channel. For an intermediate mass Higgs, the dilepton final state $H \rightarrow W W^{(*)} \rightarrow l \nu l \nu$ is slightly favored with respect to the $H \rightarrow W W^{(*)} \rightarrow l \nu j j$ channel because of the $W+n j$ background which affects the latter. In the first case the main background comes from $t \bar{t}$ production followed in importance by EW $W W j j$ production which is estimated to be about $10 \%$ of the signal. In the second case the main background comes from $W+$ $n j$ followed by $t \bar{t}$ and EW $W W j j$ production. QCD $W W j j$ production can be reduced to be of the same order of magnitude as the EW contribution using acceptance cuts and can be rendered essentially negligible by a central jet veto which is not so effective in the EW case $[13,36]$.

The production channel $q q \rightarrow q q H, H \rightarrow Z Z \rightarrow l^{+} l^{-} j j$ has been examined in [40] while the channels $q q \rightarrow q q H$, $H \rightarrow Z Z \rightarrow l^{+} l^{-} \nu \bar{\nu}$, and $Z Z \rightarrow l^{+} l^{-} l^{+} l^{-}$have been considered in [39]. The $l^{+} l^{-} j j$ and $l^{+} l^{-} l^{+} l^{-}$channels are particularly interesting because they allow a direct reconstruction of the Higgs mass which in $l \nu j j$ final states must be extracted from the transverse mass distribution.

As an illustration of PHANTOM capabilities, the four body invariant mass distribution of the $\mu^{+} \mu^{-}$pair and the two most central quarks in $4 q \mu^{+} \mu^{-}$final states in the neighborhood of the Higgs peak is shown in Fig. 7 for $M(H)=150 \mathrm{GeV}$ and $M(H)=200 \mathrm{GeV}$. The plot on the

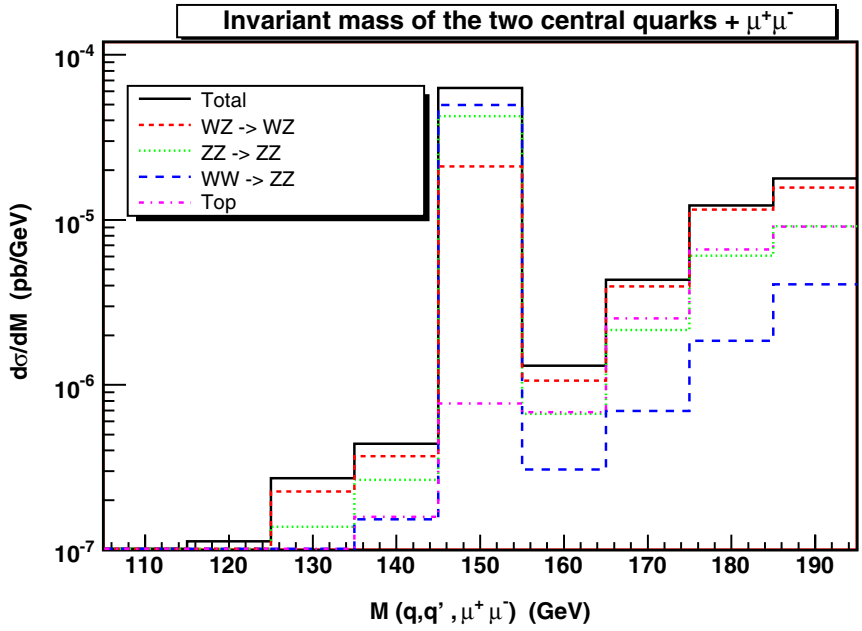

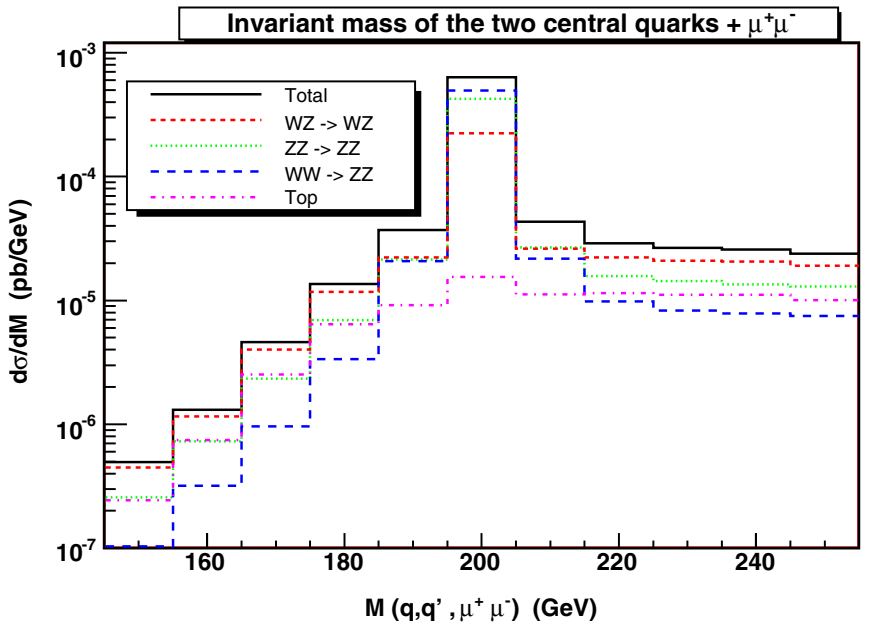

FIG. 7 (color online). Distribution of the invariant mass $M(V Z)$ of the two candidate vector bosons for a Higgs mass of $150 \mathrm{GeV}$ and $200 \mathrm{GeV}$. The contribution of the various subprocesses is evaluated as in Sec. III. 
left is obtained by zooming into the area around the Higgs peak in Fig. 6. Both plots show the results for all reactions which contain the different subprocesses, as described in Sec. III, as well as the total distribution. Assuming a mass resolution of $\pm 10 \mathrm{GeV}$ around the peak, the EW irreducible background amounts to about 3\% (13\%) for $M(H)=$ $150(200) \mathrm{GeV}$. An order of magnitude assessment of the statistical significance of such a cross section for Higgs discovery can be obtained by comparison with the $j j l \nu$ channel. The main reducible background is QCD $V+n j$ production. Assuming that the effect of acceptance cuts is similar in the $W W$ and in the $Z Z$ channels, one can estimate the ratio of the significancies $S$ in the two cases as

$$
\begin{aligned}
\frac{S\left(l^{+} l^{-} j j\right)}{S(l \nu j j)} \approx & \frac{\sigma_{q q H} \cdot B R(H \rightarrow Z Z) \cdot B R\left(Z Z \rightarrow l^{+} l^{-} j j\right)}{\sigma_{q q H} \cdot B R(H \rightarrow W W) \cdot B R(W W \rightarrow l \nu j j)} \\
& \times \frac{\sqrt{\sigma_{l \nu 4 j}}}{\sqrt{\sigma_{l^{+} l^{-}} 4 j}} .
\end{aligned}
$$

Since $\quad \sigma_{l \nu 4 j} / \sigma_{l^{+} l^{-} 4 j} \approx 10 \quad[25] \quad$ and $B R(Z Z \rightarrow$ $\left.l^{+} l^{-} j j\right) / B R(W W \rightarrow l \nu j j) \approx 1 / 3$, we are left with

$$
\frac{S\left(l^{+} l^{-} j j\right)}{S(l \nu j j)} \approx \frac{B R(H \rightarrow Z Z)}{B R(H \rightarrow W W)} .
$$

For $M(H)>200 \mathrm{GeV}$ the ratio of the two branching ratios is about 0.5 , and on the basis of the studies of the ATLAS [36] and CMS [39] collaborations for the $W W$ channel, one expects a good statistical significance, of order 5, for $q q H, H \rightarrow Z Z, Z Z \rightarrow l^{+} l^{-} j j$. This naive estimate is in rough agreement with the analysis of [40] which obtains significancies slightly below 4 in the mass range $200<M(H)<300 \mathrm{GeV}$.

Below the $Z Z$ threshold, the Higgs branching ratio to $Z Z$ reaches about 0.08 at $M(H) \approx 150 \mathrm{GeV}$. For a luminosity of $30 \mathrm{fb}^{-1}$ about 40 events are expected in the $H \rightarrow$ $l^{+} l^{-} j j, l=e, \mu$ channel. Only a complete analysis including all backgrounds and full detector simulation could tell whether this is enough for establishing a Higgs signal in this range of masses in the vector fusion channel.

\section{B. The high $V V$ mass region}

In the absence of firm predictions in the strong scattering regime, trying to gauge the possibilities of discovering signals of new physics at the LHC requires the somewhat arbitrary definition of a model of $V_{L} V_{L}$ scattering beyond the boundaries of the SM. Some of these models predict the formation of spectacular resonances which will be easily detected. For some other set of parameters in the models, only rather small effects are expected; see for instance $[8,9]$.

The simplest approach is to consider the SM in the presence of a very heavy Higgs. While this entails the violation of perturbative unitarity, the linear rise of the cross section with the invariant mass squared in the hard
$V V$ scattering will be masked by the decrease of the parton luminosities at large momentum fractions and, as a consequence, will be particularly challenging to detect. At the LHC, the offshellness of the incoming vector bosons will further increase the difference between the expectations based on the behavior of on shell $V V$ scattering and the actual results. For $M_{H}>10 \mathrm{TeV}$, all Born diagrams with Higgs propagators become completely negligible in the Unitary gauge, and the predictions for all processes in Eq. (2) reduce to those in the $M_{H} \rightarrow \infty$ limit. In this section we compare this minimalistic definition of physics beyond the standard model, which we call the no-Higgs scenario, with the predictions of the SM with a light Higgs.

An analysis of selection cuts capable of increasing the difference between the no-Higgs and light Higgs cases could provide some guidance for the search of signals of new physics in boson boson scattering.

As already mentioned, in the absence of the Higgs, all SM scattering processes between on shell weak vector bosons grow linearly with the center of mass energy squared, with the exception of $Z Z \rightarrow Z Z$. Therefore all possible reactions, $Z W \rightarrow Z W, W W \rightarrow Z Z, Z Z \rightarrow Z Z$, should be carefully investigated.

An interesting possibility is to investigate whether or not there exists an elementary Higgs boson by measuring the $V V$ cross section at large $M(V V)$. Previous studies [14] have shown that kinematical distributions are quite insensitive to the value of the Higgs mass provided it is much smaller than the invariant mass of the $V V$ system.

In order to isolate the $V V$ fusion signal, and more generally two vector boson production, from all other six fermion final state processes and investigate EWSB, differ-

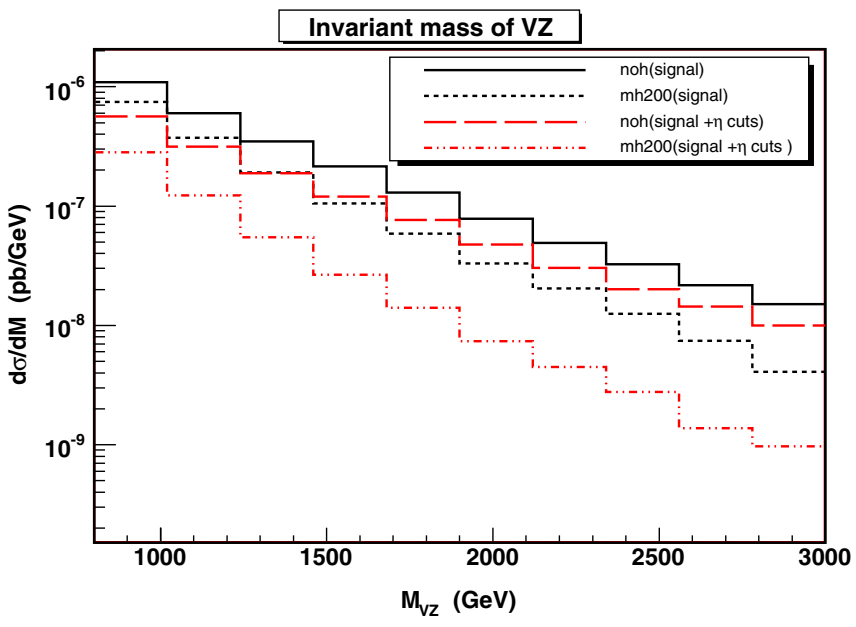

FIG. 8 (color online). Invariant mass distribution for $M(V Z)>$ $800 \mathrm{GeV}$. The solid and long-dashed lines refer to the no-Higgs case, the short-dashed and dot-dashed ones to $M(H)=$ $200 \mathrm{GeV}$. All results satisfy the standard acceptance cuts. The solid and short-dashed lines present the results for our signal definition. For the long-dashed and dot-dashed histograms, we have further required $\left|\eta\left(Z_{l l}\right)\right|<2$ and $\left|\eta\left(q_{V}\right)\right|<2$. 


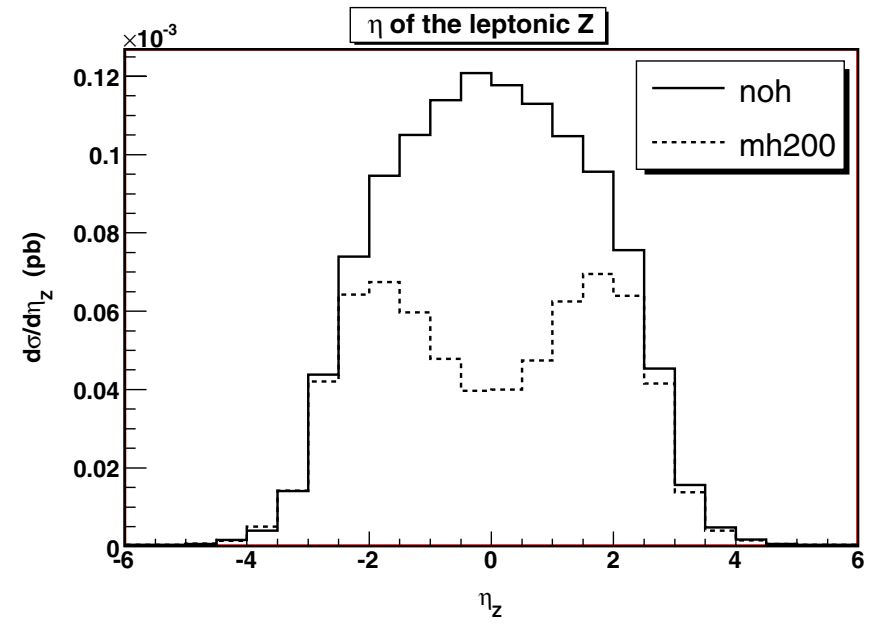

FIG. 9 (color online). Pseudorapidity distribution of the lepton pair for the no-Higgs case (solid line) and for $M(H)=200 \mathrm{GeV}$ (dashed line). All events satisfy $M(Z V)>800 \mathrm{GeV}$.

ent kinematical cuts have been applied to the simulated events.

First of all, single top production is vetoed as discussed in Sec. III. Second, the invariant mass of the two charged leptons has to reconstruct the mass of a $Z$, and is required to be in the range $M_{Z} \pm 10 \mathrm{GeV}$. In $V V$ fusion an additional $W$ or $Z$ decaying hadronically is expected to be present. Therefore events are required to contain two quarks with the correct flavor content to be produced in $W$ or $Z$ decay, with an invariant mass of $\pm 10 \mathrm{GeV}$ around the central value of the appropriate $\mathrm{EW}$ boson. If more than one combination of two quarks satisfies these requirements, the one closest to the corresponding central mass value is selected. In the following, this combination will be assumed to originate from the decay of an EW vector boson.

In a third step, in order to reject events which can be identified with the production of three vector bosons, the flavor content and the invariant mass of the two remaining quarks are compared with a $W$ and a $Z$. If compatible within $10 \mathrm{GeV}$ with either, the event is rejected. The events satisfying all these constraints will constitute the "signal" sample.

In Fig. 8 we present the invariant mass distribution of the two charged leptons and the two jets associated with the vector boson decay for $M(H)=200 \mathrm{GeV}$ and for the noHiggs case. A number of selection cuts have been studied in order to increase the difference between the two Higgs hypotheses. Simple requirements of centrality of the lepton pair and of the candidate second vector boson have proved to be the most effective. The pseudorapidity distribution of the charged lepton pair in the two cases is shown in Fig. 9. The long-dashed dot-dashed distributions in Fig. 8 have been obtained with the additional constraints that $\left|\eta\left(Z_{l l}\right)\right|<2$ and $\left|\eta\left(q_{V}\right)\right|<2$, where $q_{V}$ refers to the quarks which are associated with the vector boson decay. The corresponding distributions for the $Z W$ and $Z Z$ final states are presented in Fig. 10. The cross section for $q q Z W$ and $q q Z Z$ production are similar; however the discrepancy between the no-Higgs case and the $M(H)=200 \mathrm{GeV}$ is larger for the $q q Z Z$ final state.

In the upper part of Table $\mathrm{V}$ we present the number of events as a function of the minimum invariant mass of the
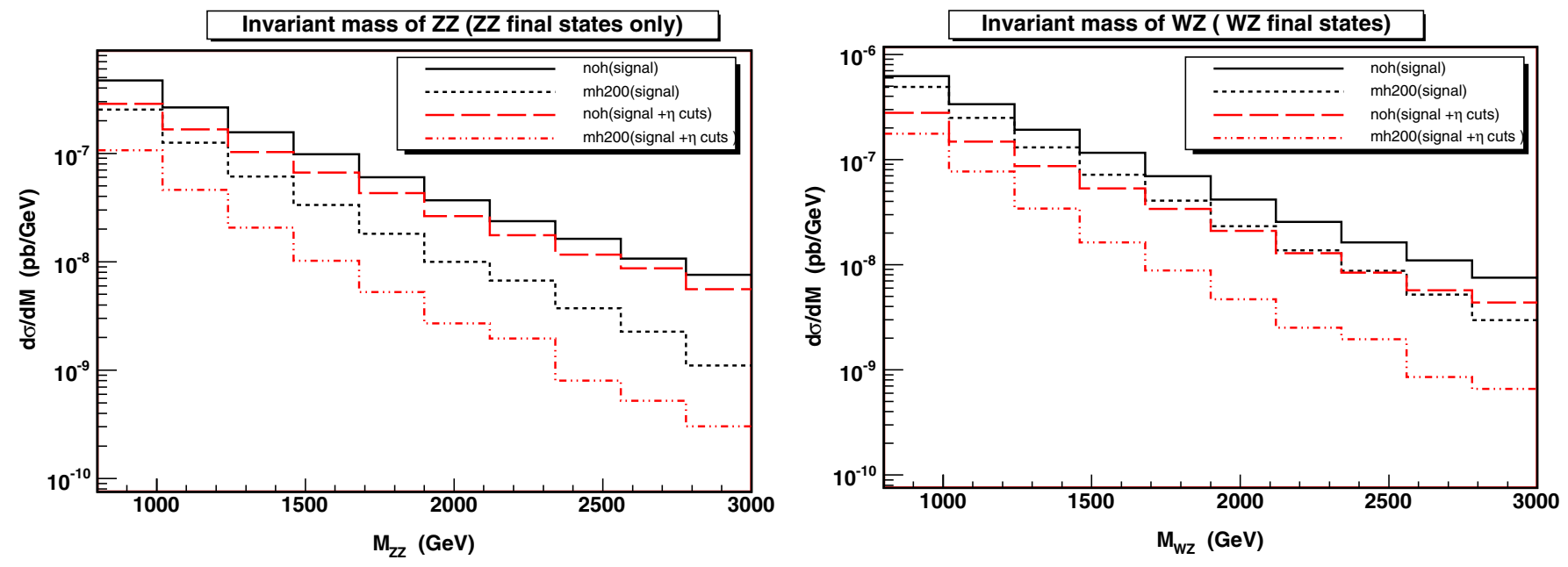

FIG. 10 (color online). $\quad M(Z Z)$ (left panel) and $M(W Z)$ (right panel) invariant mass distribution for $M(V Z)>800 \mathrm{GeV}$. The solid and long-dashed lines refer to the no-Higgs case, the short-dashed and dot-dashed ones to $M(H)=200 \mathrm{GeV}$. The solid and shortdashed lines present the results for our signal definition. For the long-dashed dot-dashed histograms, we have further required $\left|\eta\left(Z_{l l}\right)\right|<2$ and $\left|\eta\left(q_{V}\right)\right|<2$. 
TABLE V. Number of events as a function of the minimum invariant mass of the $Z V \rightarrow \mu^{+} \mu^{-} j j$ pair for $L=100 \mathrm{fb}^{-1}$. All events satisfy $\left|\eta\left(Z_{l l}\right)\right|<2$ and $\left|\eta\left(q_{V}\right)\right|<2$. In brackets we show the contribution of the $(Z W, Z Z)$ final states.

\begin{tabular}{cccccc}
\hline \hline$M_{\text {cut }}$ & \multicolumn{2}{c}{ No Higgs } & \multicolumn{2}{c}{$M(H)=200 \mathrm{GeV}$} & Ratio \\
\hline $800 \mathrm{GeV}$ & 31 & $(14,17)$ & 12 & $(7,5)$ & 2.59 \\
$900 \mathrm{GeV}$ & 25 & $(12,13)$ & 8 & $(5,3)$ & 3.12 \\
$1.0 \mathrm{TeV}$ & 19 & $(9,10)$ & 6 & $(4,2)$ & 3.16 \\
$1.1 \mathrm{TeV}$ & 16 & $(7,9)$ & 5 & $(3,2)$ & 3.20 \\
$1.2 \mathrm{TeV}$ & 13 & $(6,7)$ & 3 & $(2,1)$ & 4.33 \\
$1.3 \mathrm{TeV}$ & 11 & $(5,6)$ & 2 & $(1,1)$ & 5.50 \\
$1.4 \mathrm{TeV}$ & 9 & $(4,5)$ & 2 & $(1,1)$ & 4.50 \\
& \multicolumn{5}{c}{$\Delta R=0.4$} \\
$M_{\text {cut }}$ & No Higgs & $M(H)=200 \mathrm{GeV}$ & Ratio \\
$800 \mathrm{GeV}$ & 18 & $(8,10)$ & 10 & $(6,4)$ & 1.80 \\
$900 \mathrm{GeV}$ & 12 & $(5,7)$ & 6 & $(4,2)$ & 2.00 \\
$1.0 \mathrm{TeV}$ & 8 & $(4,4)$ & 4 & $(2,2)$ & 2.00 \\
$1.1 \mathrm{TeV}$ & 5 & $(2,3)$ & 3 & $(2,1)$ & 1.60 \\
& \multicolumn{5}{c}{$\Delta R=0.5$} \\
$M_{\text {cut }}$ & No Higgs & $M(H)=200 \mathrm{GeV}$ & Ratio \\
$800 \mathrm{GeV}$ & 12 & $(5,7)$ & 8 & $(5,3)$ & 1.50 \\
$900 \mathrm{GeV}$ & 8 & $(4,4)$ & 5 & $(3,2)$ & 1.60 \\
$1.0 \mathrm{TeV}$ & 5 & $(2,3)$ & 3 & $(2,1)$ & 1.60 \\
\hline \hline
\end{tabular}

$\mu^{+} \mu^{-} j j$ system for $L=100 \mathrm{fb}^{-1}$ with the set of cuts shown in Table II. In brackets we also give the separate results for the $Z W$ and $Z Z$ final states. The number of events is smaller than the expected yield in the $4 q \mu \nu$ channel [14] but the differences between the two Higgs hypotheses are larger. In fact, similar ratios are obtained with a comparable number of events.

In Table $\mathrm{V}$ we also show the effect of requiring a minimum $\Delta R$ separation among colored partons. The ex- pected number of events decreases sharply, by about a factor of 2 for $\Delta R=0.4$ and by about a factor of 3 for $\Delta R=0.5$, in the no-Higgs case. The statistics for a light Higgs is less affected because the vector boson distribution is less central in this case and it is precisely the jets originating from the most central and higher $p_{T} W$ 's and $Z$ 's which are most likely to merge into one jet under the effect of a $\Delta R$ cut. As already mentioned in Sec. III, this issue is well known and various approaches have been tried in the literature. ATLAS [3] (Secs. 9.3.1.3, 19.2.10.2) favors using a small cone $\Delta R=0.2$ for the determination of the jet centroid and then a larger cone $\Delta R=0.4$ for collecting the energy flow of the jets. In QCD studies at the LHC a typical separation $\Delta R=0.5$ is adopted. In Refs. [9,41] a different approach has been proposed. First, jets with a total invariant mass in the neighborhood of the electroweak vector meson mass are selected. Then these jets are forced to divide into two subjets, by varying the separation parameter $y$ in the $k_{\perp}$ scheme. For jets originating from a vector meson decay, the scale $\sqrt{y p_{T}^{2}}$ at which the heavy jet splits into two subjets is typically close to the vector mass, while for standard QCD jets the splitting scale is much smaller. The subject of jet reconstruction algorithms is still lively debated. Since EW vector bosons are crucial to many investigations at the LHC, we expect that a scheme which allows one to distinguish jets produced in the decay of high $p_{T} W$ 's and $Z$ 's will be devised for this kind of specialized study.

At the LHC, the expected mass resolution for vector bosons decaying to dijets is about $5 \div 10 \mathrm{GeV}$, depending on the boson transverse momentum [3]. This makes it quite difficult to separate $Z Z \rightarrow \mu^{+} \mu^{-} j j$ from $Z W \rightarrow \mu^{+} \mu^{-} j j$ final states on the basis of the invariant mass of the jet pair. It is therefore of interest to explore alternative means of separating the two final states. The low energy theorem
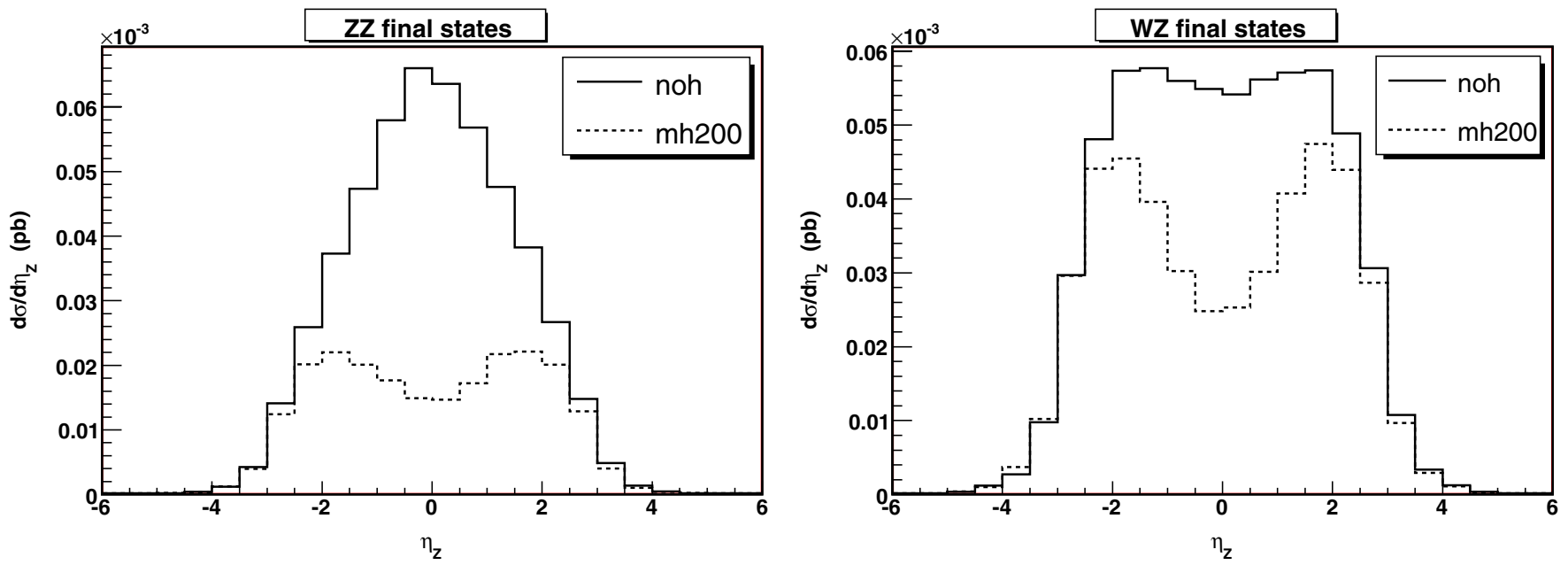

FIG. 11 (color online). Pseudorapidity distribution of the lepton pair in $q q Z Z$ (left panel) and $q q Z W$ (right panel) final states. The solid line refers to the no-Higgs case, the dashed one to $M(H)=200 \mathrm{GeV}$. In all cases $M(V Z)>800 \mathrm{GeV}$. 
[38] predicts that $A\left(W^{+} W^{-} \rightarrow Z Z\right)=s / v^{2}$, where $A$ is the scattering amplitude, $s$ is the usual Mandelstam variable, and $v$ is the coupling strength of the gauge current to the Goldstone boson, which in the SM coincides with the vacuum expectation value of the Higgs field. As a consequence of crossing symmetry then $A(W Z \rightarrow W Z)=t / v^{2}$. Therefore, averaging between the two orientations of the incoming $Z W$ system, and neglecting vector boson masses, one expects, in the reaction center of mass, $d \sigma / d \cos \theta \sim$ const for $W^{+} W^{-} \rightarrow Z Z$ and $d \sigma / d \cos \theta \sim\left(1+\cos \theta^{2}\right)$ for $W Z \rightarrow W Z$ where $\theta$ is the scattering angle in the boson boson center of mass. This has led us to study the pseudorapidity distribution of the lepton pair in $q q Z Z$ and $q q Z W$ final states separately, as shown in Fig. 11. Despite the fact that we are not in the center of mass of the $V Z$ system, that the incoming vector bosons are not on their mass shell, and that we are plotting the distribution of an angular variable which is not the cosine of the center of mass scattering angle, the general prediction that the two final states have different distributions is verified. In the no-Higgs case, the $Z W$ channel distribution is almost flat in the region $|\eta|<2$ with small peaks in the forward and backward directions, as suggested by the LET, while the $Z Z$ one peaks at zero rapidity. It is somewhat surprising, but quite welcome, that the two distributions are markedly different also when a light Higgs boson is present in the spectrum as expected in the SM. It should be mentioned that the angular distribution depends on the vector boson pair invariant mass. The plot for $M(V Z)>300 \mathrm{GeV}$, which we do not show, displays a similar, rather central, behavior for the two processes.

The selection procedure employed for Fig. 8 and Table V is not fully realistic: no flavor information will be available for light quarks and $b$ 's will be tagged only in the central part of the detector. At this stage we want to isolate as much as possible the $V V$ fusion signal from all other production channels, with the aim of identifying a possible signal definition which could play the role which was played by CC03 at LEP2 [42], capturing the essence of the physical phenomena under investigation and allowing comparisons between the results from different collaborations. It is however of interest to investigate whether the relevant distributions are sensitive to the details of the selection cuts. In Fig. 12 we compare the invariant mass distribution of the two charged leptons and the two quarks associated with the vector boson obtained with two different methods. In the first case (dotted line) we select only quark pairs that have the correct flavor content to be produced in $W$ or $Z$ decay, while in the second (dot-dashed line) we consider all quark pairs. In the more realistic setting the top veto is applied to any triplet of quarks with a total invariant mass between 160 and $190 \mathrm{GeV}$; moreover all events in which two quark pairs with mass between $M_{W}-10 \mathrm{GeV}$ and $M_{Z}+10 \mathrm{GeV}$ are present are discarded. In both cases we identify the candidate vector

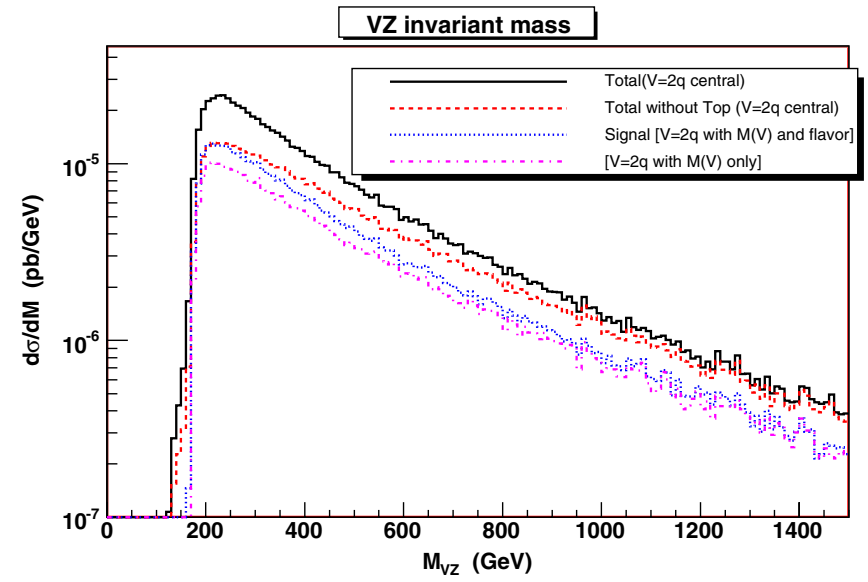

FIG. 12 (color online). Invariant mass distribution of the lepton pair and the two jets from boson decay for the no-Higgs case. The solid (dashed) line is obtained by identifying the two most central jets as the vector boson decay products before (after) top vetoing. The dotted line is obtained by requiring the correct flavor content for the jets identified as decay products of both the vector boson and the top. The dot-dashed line is produced using solely invariant mass information to identify the vector boson and the top decay products.

boson with the quark pair whose mass is closest to the nominal vector boson mass. The two distributions differ by about $20 \%$ at small invariant masses but agree quite nicely at invariant masses above $800 \mathrm{GeV}$, showing that our results based on Monte Carlo level flavor information are not seriously degraded when selection procedures closer to the actual experimental practice are adopted. For comparison, we also present the invariant mass distribution obtained by identifying the two most central jets as the vector boson decay products before (solid line) and after (dotted line) top vetoing.

\section{CONCLUSIONS}

In this paper we have studied all $q_{1} q_{2} \rightarrow q_{3} q_{4} q_{5} q_{6} l^{+} l^{-}$ processes at order $\mathcal{O}\left(\alpha_{e m}^{6}\right)$ at the LHC using for the first time a full fledged six fermion Monte Carlo event generator. We have studied Higgs production and its irreducible EW background in vector boson fusion followed by the decay chain $H \rightarrow Z Z \rightarrow l^{+} l^{-} j j$, including exactly all electroweak irreducible backgrounds and, in particular, the interference of $\mathrm{EW} Z Z+2 j$ and $Z W+2 j$ production with the signal. The EW irreducible background in the neighborhood of the Higgs peak amounts to about $1.5 \%$ $(6 \%)$ for $M(H)=150(200) \mathrm{GeV}$. We have examined how simple kinematical cuts can be applied at generator level to extract the $V V$ signal from the irreducible background. In the high mass region we have compared the case of a relatively light Higgs with the no-Higgs case. The integrated cross section for the latter is more than twice that in the former for a minimum invariant mass of the $Z V$ pair of 
about $800 \mathrm{GeV}$. Summing up the muon and the electron channels, about 25 events are expected in the light Higgs case for $L=100 \mathrm{fb}^{-1}$. These results are encouraging and show that a more complete analysis, including all QCD backgrounds and an accurate study of jet separation algorithms in the high invariant mass region, is worthwhile.

\section{ACKNOWLEDGMENTS}

E. A. is supported by the Italian Ministero dell'Istruzione, dell'Università e della Ricerca (MIUR) under Contract Decreto MIUR 26-01-2001 N.13 "Incentivazione alla mobilità di studiosi stranieri ed italiani residenti all'estero." Work supported by MIUR under Contract No. 2004021808_009.
[1] Proceedings of the Large Hadron Collider Workshop, Aachen, 1990, edited by G. Jarlskog and D. Rein (CERN Report No. 90-10).

[2] A. Djouadi, arXiv:hep-ph/0503172.

[3] ATLAS Collaboration, CERN Technical Design Report No. CERN-LHCC-99-14 and No. CERN-LHCC-99-15.

[4] K. A. Assamagan, M. Narain, A. Nikitenko, M. Spira, and D. Zeppenfeld (conv.) et al. (Higgs Working Group), in Proceedings of Les Houches Workshop on Physics at TeV Colliders, France, 2003 (arXiv:hep-ph/0406152).

[5] ALEPH, DELPHI, L3, and OPAL Collaborations, LEP Electroweak Working Group, and SLD Heavy Flavour Group, arXiv:hep-ex/0412015; http://lepewwg.web.cern. ch/LEPEWWG.

[6] ALEPH, DELPHI, L3, and OPAL Collaborations, and LEP Electroweak Working Group for Higgs Boson Searches, Phys. Lett. B 565, 61 (2003).

[7] M.S. Chanowitz, arXiv:hep-ph/9812215.

[8] J. Bagger et al., Phys. Rev. D 52, 3878 (1995); A. Dobado, M. J. Herrero, J. R. Peláez, and E. Ruiz Morales, Phys. Rev. D 62, 055011 (2000).

[9] J. M. Butterworth, B. E. Cox, and J. R. Forshaw, Phys. Rev. D 65, 096014 (2002).

[10] M. J. Duncan, G. L. Kane, and W. W. Repko, Nucl. Phys. B272, 517 (1986); D. A. Dicus and R. Vega, Phys. Rev. Lett. 57, 1110 (1986); J. F. Gunion, J. Kalinowski, and A. Tofighi-Niaki, Phys. Rev. Lett. 57, 2351 (1986).

[11] R. N. Cahn, S. D. Ellis, R. Kleiss, and W. J. Stirling, Phys. Rev. D 35, 1626 (1987); V. Barger, T. Han, and R. Phillips, Phys. Rev. D 37, 2005 (1988); 36, 295 (1987); R. Kleiss and J. Stirling, Phys. Lett. B 200, 193 (1988); V. Barger et al., Phys. Rev. D 42, 3052 (1990); 44, 1426 (1991); 46, 2028 (1992); D. Froideveaux, in Ref. [1], Vol. II, p. 444; M. H. Seymour, in Ref. [1], p. 557; U. Baur and E. W. N. Glover, Phys. Lett. B 252, 683 (1990); D. Dicus, J. Gunion, and R. Vega, Phys. Lett. B 258, 475 (1991); D. Dicus, J. Gunion, L. Orr, and R. Vega, Nucl. Phys. B377, 31 (1992); U. Baur and E. W. N. Glover, Nucl. Phys. B347, 12 (1990).

[12] J. Bagger et al., Phys. Rev. D 49, 1246 (1994); V. Barger, R. Phillips, and D. Zeppenfeld, Phys. Lett. B 346, 106 (1995); J. Bagger et al., Phys. Rev. D 52, 3878 (1995); K. Iordanidis and D. Zeppenfeld, Phys. Rev. D 57, 3072 (1998).

[13] D. Rainwater and D. Zeppenfeld, Phys. Rev. D 60, 113004 (1999); 61, 099901(E) (2000); D. Rainwater, arXiv:hep$\mathrm{ph} / 9908378$.
[14] E. Accomando, A. Ballestrero, S. Bolognesi, E. Maina, and C. Mariotti, J. High Energy Phys. 03 (2006) 093.

[15] O. J. P. Eboli, M. C. Gonzalez-Garcia, and J. K. Mizukoshi, Phys. Rev. D 74, 073005 (2006).

[16] M.S. Chanowitz and M. K. Gaillard, Nucl. Phys. B261, 379 (1985); Phys. Lett. 142B, 85 (1984), and Ref. [1]; G. Kane, W. Repko, and B. Rolnick, Phys. Lett. 148B, 367 (1984); S. Dawson, Nucl. Phys. B249, 42 (1985).

[17] E. Accomando, A. Ballestrero, A. Belhouari, and E. Maina, Phys. Rev. D 74, 073010 (2006).

[18] E. Accomando, A. Ballestrero, and E. Maina, J. High Energy Phys. 07 (2005) 016.

[19] E. Accomando, A. Ballestrero, and E. Maina, Nucl. Instrum. Methods Phys. Res., Sect. A 534, 265 (2004); Proceedings of the 18th International Workshop on HighEnergy Physics and Quantum Field Theory (QFTHEP 2004), St. Petersburg, Russia, 2004 (arXiv:hep-ph/ 0505225).

[20] A. Ballestrero, A. Belhouari, G. Bevilacqua, and E. Maina (unpublished).

[21] A. Bredenstein, A. Denner, S. Dittmaier, and M.M. Weber, Phys. Rev. D 74, 013004 (2006); J. High Energy Phys. 02 (2007) 080; A. Denner, S. Dittmaier, M. Roth, and M. M. Weber, Nucl. Phys. B660, 289 (2003).

[22] E. Accomando (unpublished).

[23] A. Ballestrero and E. Maina, Phys. Lett. B 350, 225 (1995).

[24] A. Ballestrero, in Proceedings of the 14th International Workshop on High Energy Physics and Quantum Field Theory (QFTHEP 99), edited by B. B. Levchenko and V. I. Savrin (SINP MSU, Moscow, 1999), p. 303 [arXiv:hep$\mathrm{ph} / 9911318]$.

[25] M.L. Mangano, M. Moretti, F. Piccinini, R. Pittau, and A. D. Polosa, J. High Energy Phys. 07 (2003) 001.

[26] S. R. Slabospitsky and L. Sonnenschein, Comput. Phys. Commun. 148, 87 (2002).

[27] F. Krauss, R. Kühn, and G. Soff, J. High Energy Phys. 02 (2002) 044; A. Schalicke, F. Krauss, R. Kühn, and G. Soff, J. High Energy Phys. 12 (2002) 013.

[28] E. E. Boos, M. N. Dubinin, V. A. Ilyin, A. E. Pukhov, and V. I. Savrin, arXiv:hep-ph/9503280; A. Pukhov et al., arXiv:hep-ph/9908288.

[29] T. Ishikawa et al. (Minami-Tateya Collaboration), KEK Report No. KEK-92-19; H. Tanaka et al. (Minami-Tateya Collaboration), Nucl. Instrum. Methods Phys. Res., Sect. A 389, 295 (1997); F. Yuasa et al., Prog. Theor. Phys. Suppl. 138, 18 (2000); S. Tsuno, K. Sato, J. Fujimoto, T. 
Ishikawa, Y. Kurihara, S. Odaka, and T. Abe, Comput. Phys. Commun. 151, 216 (2003).

[30] F. Maltoni and T. Stelzer, J. High Energy Phys. 02 (2003) 027; T. Stelzer and W. F. Long, Comput. Phys. Commun. 81, 357 (1994); H. Murayama, I. Watanabe, and K. Hagiwara, KEK Report No. KEK-91-11.

[31] C. G. Papadopoulos, Comput. Phys. Commun. 137, 247 (2001); A. Kanaki and C. G. Papadopoulos, Comput. Phys. Commun. 132, 306 (2000).

[32] M. Moretti, T. Ohl, and J. Reuter, arXiv:hep-ph/0102195; W. Kilian, 2nd ECFA/DESY Study 1998-2001, LC-TOOL2001-039, 2001, pp. 1924-1980.

[33] B. Jäger, C. Oleari, and D. Zeppenfeld, J. High Energy Phys. 07 (2006) 015.

[34] B. Jäger, C. Oleari, and D. Zeppenfeld, Phys. Rev. D 73, 113006 (2006).
[35] T. Binoth, M. Ciccolini, N. Kauer, and M. Kramer, J. High Energy Phys. 03 (2005) 065.

[36] S. Asai et al., Eur. Phys. J. C 32S2, s19 (2004).

[37] H. L. Lai et al. (CTEQ Collaboration), Eur. Phys. J. C 12, 375 (2000).

[38] S. Weinberg, Phys. Rev. Lett. 17, 616 (1966); M. S. Chanowitz, M. Golden, and H. M. Georgi, Phys. Rev. D 36, 1490 (1987); Phys. Rev. Lett. 57, 2344 (1986).

[39] S. Abdulin et al., CMS Note 2003/033.

[40] K. Crammer et al., arXiv:hep-ph/0401148.

[41] J. M. Butterworth, J. Ellis, and A. R. Raklev, arXiv:hep$\mathrm{ph} / 0702150$.

[42] M.W. Grünewald et al., Four Fermion Working Group Report of the LEP2 MC Workshop, CERN Report No. 2000-009, 2000. 